\title{
A Dietary Supplement with a High Eicosapentaenoic Acid to Docosahexaenoic Acid Ratio Reduces Triglyceride Levels in Mildly Hypertriglyceridemic Subjects
}

\author{
Alan S. Ryan, Stephen S. Porter, Frederick D. Sancilio \\ Department of Clinical Research, Sancilio and Company, Riviera Beach, USA. \\ Email: alryan@sancilio.com \\ Received November $14^{\text {th }}, 2012$; revised December $14^{\text {th }}, 2012$; accepted December $22^{\text {nd }}, 2012$
}

\begin{abstract}
There is compelling evidence that omega-3 long-chain polyunsaturated fatty acids (n-3 PUFA), eicosapentaenoic acid (EPA, 20:5n-3) and docosahexaenoic acid (DHA, 22:6n-3) provide cardioprotective benefits. This open-label study evaluated whether an omega-3 fatty acid dietary supplement with a high EPA to DHA ratio (2.3:1) reduces triglyceride (TG) levels in mildly hypertriglyceridemic subjects. Twenty subjects, with a mean baseline TG level of $321.7 \pm 108.7$ $\mathrm{mg} / \mathrm{dL}$, were administered $4 \mathrm{~g} /$ day of Ocean Blue ${ }^{\mathbb{B}}$ Professional Omega-3 $2100^{\mathrm{TM}}(\mathrm{OB})$ supplements. Each gram of OB contains the ethyl esters of EPA $(675 \mathrm{mg})$ and DHA $(300 \mathrm{mg})$. Baseline and end of study blood values were collected to assess changes in fasting levels of TG, total cholesterol (TC), LDL-cholesterol (LDL-C), and HDL-cholesterol (HDL-C). Supplementation was provided for 30 to 228 days (mean $=106 \pm 50$ days). Mean age at enrollment was 50.3 \pm 6.3 years. Compared with baseline values, mean TG levels decreased by $48 \%(p=0.001)$. There were no changes in TC and HDL-C levels $(p=$ NS); however, subjects had a significant increase in LDL-C levels $(16.4 \%, p=0.05)$. Results indicated that a high ratio of EPA to DHA $(>2: 1)$ had a statistically significant TG-lowering effect in mildly hypertriglyceridemic subjects. The lipid effects of $\mathrm{OB}$ are compared with those published in the literature for $\mathrm{n}-3$ drugs indicated for hypertriglyceridemia (very high TG levels, $>500 \mathrm{mg} / \mathrm{dL}$ )
\end{abstract}

Keywords: Omega-3 Fatty Acids; Dietary Supplements; Lipid Lowering Medications

\section{Introduction}

The cardiovascular benefits of the omega- 3 long-chain polyunsaturated fatty acids (n-3 PUFA), eicosapentaenoic acid (EPA, 20:5n-3) and docosahexaenoic acid (DHA, 22:6n-3), are well established [1-3]. Large-scale epidemiological studies have demonstrated an inverse relationship between the intake of n-3 PUFA and coronary heart disease (CHD) mortality [4-6]. The cardioprotective effect of n-3 PUFA is especially strong among high-risk individuals who are hypercholesterolemic or had a previous myocardial infarction $[7,8]$.

Since the availability of purified forms of EPA and DHA in the marketplace, researchers have started to investigate the differential effects that EPA and DHA have on heart health $[9,10]$. Products containing both EPA and DHA not only lower triglyceride (TG) levels but also decrease the growth rate of atherosclerotic plaque, improve endothelial function, and reduce inflammatory response [2]. Individually, EPA and DHA lower TG levels, and favorably affect arterial compliance, measures of inflammation, and oxidative stress [10]. While only DHA appears to reduce heart rate and blood pressure and increase HDL levels and LDL particle size [10], purified EPA is associated with the reduced risk of nonfatal coronary endpoints in a large clinical trial [8].

In a recent scientific statement from the American Heart Association (AHA) an elevated TG level is directly associated with increased atherosclerotic risk [1]. TG levels serve as an important serum biomarker for CHD, especially combined with low HDL-cholesterol (HDL-C) and elevated LDL-cholesterol (LDL-C) levels [1]. Data from the 1999-2006 National Health and Nutrition Examination Surveys (NHANES) indicate that $31 \%$ of the adult US population has a TG level $\geq 150 \mathrm{mg} / \mathrm{dL}$ [11]. Among US populations, Mexican-Americans have the highest rates $(34.9 \%)$ followed by non-Hispanic Whites (33\%) and Blacks (15.6\%). High (200 - $499 \mathrm{mg} / \mathrm{dL})$ and very high $(\geq 500 \mathrm{mg} / \mathrm{dL}) \mathrm{TG}$ levels were observed in $16.2 \%$ and $1.1 \%$ of US adults, respectively. Very high TG levels or hypertriglyceridemia (HTG) (TG $\geq 500$ $\mathrm{mg} / \mathrm{dL}$ ) is frequently found in combination with insulin resistance and obesity [1,2]. In addition to CHD, HTG is 
typically associated with metabolic syndrome and can cause a number of medical problems including acute pancreatitis.

Dietary intakes of n-3 PUFA are very low in most western societies. Dietary intake data from NHANES (1999-2000) indicate that fish consumption in the US population is inadequate, resulting in a mean intake of only $100 \mathrm{mg}$ /day of EPA and DHA combined [12]. Along with important lifestyle changes, including dietary changes and weight loss, the AHA recommends a daily intake of 500 - $1000 \mathrm{mg}$ of n-3 PUFA for individuals with borderline high TG (150 - $199 \mathrm{mg} / \mathrm{dL}), 1000$ - 2000 $\mathrm{mg}$ for individuals with high TG (200 - $499 \mathrm{mg} / \mathrm{dL})$, and $2000-4000 \mathrm{mg}$ for individuals with very high TG levels ( $\geq 500 \mathrm{mg} / \mathrm{dL}$ ) [1]. For individuals who prefer not to eat fatty fish such as herring, salmon, or sardines, dietary supplements of n-3 PUFA are available as a substitute.

In a small pilot study $(n=5)$ that considered $4 \mathrm{~g}$ /day of the dietary supplement Ocean Blue ${ }^{\circledR}$ Professional Omega-3 $2100^{\mathrm{TM}}(\mathrm{OB})$ which contains $675 \mathrm{mg}$ of EPA and $300 \mathrm{mg}$ DHA as ethyl esters (EPA to DHA ratio of 2.3:1) the mean TG level was reduced by $48 \%$ among individuals with a baseline mean TG level of $332.8 \pm 157.6 \mathrm{mg} / \mathrm{dL}$. The purpose of the present study was to confirm the results of the initial pilot study. We also compare the lipid effects produced by $\mathrm{OB}$, a dietary supplement with a high EPA to DHA ratio, to the published lipid data for other n-3 PUFA products with different EPA to DHA ratios. An open-label clinical trial was conducted with subjects with elevated TG levels (mean $=321.7 \pm 108.7$ $\mathrm{mg} / \mathrm{dL}$ ) who were administered $4 \mathrm{~g} /$ day of $\mathrm{OB}$ for up to 228 days.

\section{Materials and Methods}

This was an open-label study that evaluated the effects of OB on fasting serum levels of TG, TC, LDL-C and HDL-C. The trial was conducted in the private clinics of two physicians located in Roanoke, VA and Boynton Beach, FL. Upon receiving signed informed consent from interested subjects, the medical record of each was screened for study eligibility. Twenty-five adult male and females (ages $>18$ years) with TG levels above 170 $\mathrm{mg} / \mathrm{dL}$ were initially screened for study participation; twenty subjects agreed to participate (11 males, 9 females). Subjects were eligible to participate if they had a history of CHD defined as acute myocardial infarction, unstable angina, coronary artery bypass graft surgery, percutaneous coronary, smoking, or hypertension. Diabetic subjects were included in the study.

Concomitant use of statins, thiazide diuretics, betablockers, estrogen, and levothyroxine, was permitted if the doses of these medications remained unchanged during the trial. Subjects were excluded from the study if they were pregnant or breastfeeding, taking omega-3 fatty acid supplementation, consuming more than two servings of fish/week, or if they failed to provide written informed consent.

Baseline fasting lipid panel (FLP), alanine aminotransferase $(\mathrm{AL})$, creatine kinase $(\mathrm{CK})$, fasting blood glucose $(\mathrm{FBG})$, and $\mathrm{HBA}_{1 \mathrm{c}}$ levels (for subjects with diabetes mellitus) were obtained for each potential study subject.

\section{Procedures}

Patients were asked to take four capsules of OB/day. Each capsule contains $675 \mathrm{mg}$ of EPA and $300 \mathrm{mg}$ DHA with a total omega-3 fatty acid content of $1050 \mathrm{mg}$. The other inactive, small amounts $(<3.9 \%$ w/w each $)$ of omega-3 fatty acids in each capsule were stearidonic acid, 18:4n-3; eicosatetraenoic acid, 20:4n-3; heneicosapentaeonic acid, 21:5n-3; docosapentaenoic acid, 22:5n-3). Subjects were advised to take two capsules twice a day with meals. Each subject received at least a month supply of capsules and returned to the office each month for another supply. At each visit, any remaining capsules were counted to ensure compliance. Study subjects were instructed to maintain their current diet, exercise habits, and alcohol intake throughout the study period. FLP, ALT, CK, and FBG measurements were obtained for each subject monthly until the study was concluded.

We chose an open-label, non-comparator, two-site design for this nutrition study of OB capsules because the main objective was to evaluate blood biochemical markers that are routinely measured during clinic visits of subjects with CHD. Results from this study were compared with those published in the literature for other $n-3$ PUFA products.

\section{Outcome Measures}

The primary outcome measure was the change in mean TG levels from baseline to end of study. Secondary outcomes included changes in TC, LDL-C, and HDL-C levels. Safety assessments included monitoring large elevations in ALT (3 times upper limit of normal, >120 IU/L), CK (greater than upper limit of normal, $>209 \mathrm{mg} / \mathrm{dL}$ ), and FBG (greater than upper limit of normal, $>100$ $\mathrm{mg} / \mathrm{dL}$ ) levels; however, all ALT, CK, and FBG levels remained in the normal range for all subjects throughout the study.

\section{Statistical Analysis}

Subject characteristic data were evaluated at time of enrollment. Descriptive statistics were used for subject 
characteristic data with proportions and means \pm standard deviations reported where appropriate. Normality of data was tested using Shapiro Wilk's test. Paired t-test was used for normally distributed data while Wilcoxon's signed rank test was used for non-normal data to determine significant differences within groups. Twenty subjects were recruited and completed the study.

\section{Results}

\subsection{Subject Characteristics}

Male and female subjects, aged 38 to 61 years, with elevated TG levels (172 to $586 \mathrm{mg} / \mathrm{dL}$ ) were enrolled. Baseline characteristics of enrolled subjects are provided in Table 1. The mean age of study subjects was $50.3 \pm$ 6.3 years. Subjects were distributed evenly among women and men and nearly all had hypertension. Twentyfive percent were receiving statin therapy and $20 \%$ had a prior diagnosis of diabetes mellitus. The compliance rate, determined from capsule count, was $95 \%$.

\subsection{Lipid Effects}

Compared with baseline values, the mean TG levels decreased by $48 \%(p=0.001)$ (Table 2). There were no changes in TC and HDL-C levels ( $p=$ NS); however, subjects had a significant increase in LDL-C levels $(p=$ $0.05)$ compared with baseline levels. There were no reported adverse events. The supplement was well tolerated by all subjects.

\section{Discussion}

In the present study, we evaluated the effects that n-3 PUFA supplementation had on lipid levels in subjects with elevated TG levels enrolled in a small open-label clinical study. The supplement used, OB, contained a high ratio of EPA to DHA (2.3:1). The study demonstrated a mean TG reduction of $48 \%$ using $4 \mathrm{~g} /$ day of OB among subjects with TG levels ranging from 172 to 586 $\mathrm{mg} / \mathrm{dL}$ at enrollment, the majority of whom had at least one risk factor for CHD. Five of the 20 subjects were receiving statin therapy prior to study initiation.

Considering other commercially available n-3 products, Lovaza ${ }^{\circledR}$ (omega-3 acid ethyl esters, P-OM3), the first n-3 PUFA product to be approved by the US Food and Drug Administration (FDA) as a drug to treat individuals with very high TG levels $(\geq 500 \mathrm{mg} / \mathrm{dL}$ ), has a different EPA to DHA ratio (1.2:1) [13]. Each 1 gram capsule of P-OM3 contains a combination of the ethyl

Table 1. Baseline subject characteristics.

\begin{tabular}{cc}
\hline Variable & Ocean Blue $^{\circledR}$ Professional Omega-3 2100 \\
\hline Male $(\%)$ & $11(55)$ \\
Age in years $\left(\mathrm{SD}^{\mathrm{a}}\right)$ & $50.3(6.3)$ \\
\hline Duration of treatment in days (SD) & $106(50)$ \\
Cardiovascular risk factors ${ }^{\mathrm{b}}(\%)$ & \\
Hypertension & $14(70)$ \\
Diabetes mellitus & $4(20)$ \\
Current smoker & $4(20)$ \\
Concomitant statin use (\%) & $5(25)$ \\
\hline
\end{tabular}

${ }^{\mathrm{a}} \mathrm{SD}=$ standard deviation; ${ }^{\mathrm{b}}$ Data not mutually exclusive.

Table 2. Mean $\pm \mathrm{SD}^{\mathrm{a}}$ changes from baseline in plasma lipid parameters.

\begin{tabular}{|c|c|c|c|c|}
\hline \multirow{2}{*}{ Lipid parameter } & \multicolumn{4}{|c|}{ Ocean Blue $^{\circledR}$ Professional Omega-3 $2100^{\mathrm{TM}}(\mathbf{n}=20)$} \\
\hline & Baseline & End of treatment & Change from baseline (\%) & P-value ${ }^{b}$ \\
\hline Triglyceride (mg/dL) & $321.7 \pm 108.7$ & $167.2 \pm 62.0$ & $-48.0 \pm 17.3$ & 0.001 \\
\hline Total cholesterol (mg/dL) & $210.5 \pm 64.5$ & $203.4 \pm 58.6$ & $-7.1 \pm 31.1$ & 0.20 \\
\hline LDL cholesterol (mg/dL) & $110.5 \pm 57.9$ & $128.6 \pm 59.5$ & $16.4 \pm 44.3$ & 0.05 \\
\hline HDL cholesterol (mg/dL) & $39.4 \pm 6.2$ & $42.8 \pm 9.6$ & $8.6 \pm 23.1$ & 0.20 \\
\hline
\end{tabular}

${ }^{\mathrm{a}} \mathrm{SD}=$ standard deviation; ${ }^{\mathrm{b}}$ Paired t-tests, compared to baseline. 
esters of EPA (465 mg) and DHA (375 mg). The daily dose of P-OM3 is $4 \mathrm{~g} /$ day. The reported mean reduction of TG levels among adults in the clinical trials used for the drug approval of P-OM3 was $28.0 \%(\mathrm{n}=206)$ in a patient population with a comparable baseline TG level (P-OM3, $422.8 \mathrm{mg} / \mathrm{dL}$ vs. OB, $321.7 \mathrm{mg} / \mathrm{dL}$ ) [13]. Changes in TC, LDL-C, and HDL-C levels were similar between $\mathrm{OB}$ and $\mathrm{P}-\mathrm{OM} 3$. Note that the lipid data presented in the package insert (PI) for P-OM3 are different from those provided in the drug application that was the basis of FDA approval [13], the latter being the data presented in the present study. The PI considered only those patients $(\mathrm{n}=42)$ with TG levels $\geq 500 \mathrm{mg} / \mathrm{dL}$ with a median baseline of $816 \mathrm{mg} / \mathrm{dL}$.

Vascepa $^{\mathrm{TM}}$ (icosapent ethyl, AMR101), was recently approved by FDA for use in patients with severe HTG (TG $\geq 500 \mathrm{mg} / \mathrm{dL}$ ). Compared to OB and P-OM3, which contains both EPA and DHA, AMR101 contains only EPA ( $>96 \%$ as an ethyl ester). As with OB, as dosed in the present study, the daily dose of AMR101 is also 4 g/day. After 12 weeks of treatment, AMR101 reduced placebo-corrected median TG levels by $33.1 \%$ [14]. AMR101 did not significantly increase placebo-corrected median LDL-cholesterol levels. Median decrease of TG $(-34.9 \%)$ levels in the present study using OB was comparable to that reported for AMR101 (-33.1\%), while the median increase in HDL-C level was higher $(10.3 \%$ vs. $-3.5 \%)$. OB increased the median LDL-C level by $10.3 \%$. Although fish oils containing both EPA and DHA can increase LDL-C levels the increase is associated with a shift in lipoprotein particle size from the small, atherogenic to the larger more buoyant and less atherogenic particle size(s) $[15,16]$. It is possible that AMR101 did not increase LDL-C levels because $25 \%$ of the subjects in the trial were receiving statin therapy, a drug that is specifically indicated for the reduction of elevated LDLC levels.

The limitations of our study included the small sample size, variation in duration of treatment, and absence of a placebo or active comparator used as a control. Although the sample size was small, statistical significance was achieved comparing the end of study values to those at baseline. Although lipid levels are usually not prone to a placebo effect, the lack of a placebo could have introduced some bias. In the future it might be useful to include a placebo to help eliminate any potential bias possibly associated with participating in a nutritional study. Further, any comparisons with other n-3 products should be viewed with caution. Results from other studies may differ for a variety of reasons other than treatment alone including population characteristics, duration of treatment, and concomitant medications.

Elevated TG levels are often difficult to manage wi- thin a CHD or diabetic population. For those with elevated TG levels not indicated for drug therapy $(<500$ $\mathrm{mg} / \mathrm{dL}$ ), n-3 PUFA supplementation appears to be an effective option for lowering TG levels, especially in patients receiving statin therapy [17]. Combination lipidlowering therapy using statins, fibrates, and niacin is effective, but can also increase the risk for serious adverse events such as myopathy and rhabdomyolysis $[18,19]$. The use of n-3 PUFA may be cost effective for decreasing TG levels in a CHD population or diabetic population [17].

It is intriguing that different ratios of EPA to DHA may have differing TG-lowering effects. The reasons for this outcome may be due to a variety of factors including differences in EPA and DHA bioavailability, transport by the circulatory system, and metabolic pathways of ethyl esters vs. triglyceride. More research is needed to better document and understand whether different ratios of n-3 PUFAs affect lipid levels.

\section{REFERENCES}

[1] M. Miller, N. J. Stone, C. Ballantyne, V. Bittner, M. H. Criqui, H. N. Ginsberg, et al., "Triglycerides and Cardiovascular Disease: A Scientific Statement from the American Heart Association, Circulation, Vol. 123, No. 20, 2011, pp. 2292-2333. doi:10.1161/CIR.0b013e3182160726

[2] P. M. Kris-Etherton, W. S. Harris and L. J. Appel, (for the AHA Nutrition Committee), "Omega-3 Fatty Acids and Cardiovascular Disease: New Recommendations from the American Heart Association," Arteriosclerosis, Thrombosis, and Vascular Biology, Vol. 23, No. 2, 2003, pp. 151-152. doi:10.1161/01.ATV.0000057393.97337.AE

[3] W. S. Harris, "n-3 Fatty Acids and Serum Lipoproteins: Human Studies," American Journal of Clinical Nutrition, Vol. 65, Suppl. 5, 1997, pp. 1645S-1654S.

[4] C. M. Albert, C. H. Hennekens, C. J. O’Donnell, U. A. Ajani, V. J. Care, W. C. Willett, et al., "Fish Consumpion and the Risk of Sudden Cardiac Death," Journal of the American Medical Association, Vol. 279, No. 1, 1988, pp. 23-28. doi:10.1001/jama.279.1.23

[5] M. L. Daviglus, J. Stamler, A. J. Orencia, A. R. Dyer, K. Liu, P. Greenland, et al., "Fish Consumption and the 30-Year Risk of Fatal Myocardial Infarction," New England Journal of Medicine, Vol. 336, No. 15, 1997, pp. 1046-1053. doi:10.1056/NEJM199704103361502

[6] F. B. Hu, L. Bronner, W. C. Willett, et al., "Fish and Omega-3 Fatty Acid Intake and Risk of Coronary Heart Disease in Women," Journal of the American Medical Association, Vol. 287, No. 14, 2002, pp.1815-182. doi:10.1001/jama.287.14.1815

[7] Gruppo Italiano per lo Studio Della Sopravvivenza nell'Infarto Miocardico, "Dietary Supplementation with n-3 Polyunsaturated Fatty Acids and Vitamin E after Myocardial Infarction: Results of the GISSI-Prevenzione 
Trial," Lancet, Vol. 354, No. 9177, 1999, pp. 447-455. doi:10.1016/S0140-6736(99)07072-5

[8] M. Yokoyama, H. Origasa, M. Matsuzaki, et al., "Effects of Eicosapentaenoic Acid on Major Coronary Events in Hypercholesterolemic Patients (JELIS): A Randomized, Open-Label, Blinded Endpoint Analysis," Lancet, Vol. 369, No. 9567, 2007, pp. 1090-1098. doi:10.1016/S0140-6736(07)60527-3

[9] D. Mozaffarian and J. H. Wu, "(n-3) Fatty Acids and Cardiovascular Health: Are Effects of EPA and DHA Shared or Complementary," Journal of Nutrition, Vol. 142, No. 3, 2012, pp. 614S-625S. doi:10.3945/jn.111.149633

[10] S. C. Cottin, T. A. Sanders and W. L. Hall, "The Differential Effects of EPA and DHA on Cardiovascular Risk Factors," Proceedings of the Nutrition Society, Vol. 70, No. 2, 2011, pp. 215-231. doi:10.1017/S0029665111000061

[11] J. D. Cohen, M. J. Cziraky, Q. Cai, A. Wallace, T. Wasser, J. R. Crouse, et al., "30-Year Trends in Serum Lipids among United States Adults: Results from the National Health and Nutrition Examination Surveys II, III, and 1999-2006," American Journal of Cardiology, Vol. 107, No. 7, 2010, pp. 969-975. doi:10.1016/j.amjcard.2010.05.030

[12] R. B. Ervin, J. D. Wright, C. Y. Wang and J. Kennedy-Stephenson, "Dietary Intakes of Fats and Fatty Acids for the United States Population: 1990-2000," Advance Data, Vol. 8, No. 348, 2004, pp. 1-6.

[13] Federal Drug Administration, Center for Drug Evaluation and Research, "Pharmacology/Toxicology Review and Evaluation: Omacor. Application Number 21-853, 21654s016," 2004. http://www.accessdata.fda.gov/drugsatfda_docs/nda/2007 /02853s000;\%2002165s016_PharmR.pdf

[14] H. E. Bays, C. M. Ballantyne, J. J. Kastelein, J. L. Isaacsohn, R. A. Braeckman and P. N. Soni, "Eicosapen- taenoic Acid Ethyl Ester (AMR101) Therapy in Patients with Very High Triglyceride Levels (From the MultiCenter, plAcebo-controlled, Randomized, double-blINd, 12-Week Study with an Open-Label Extension [MARINE] Trial)," American Journal of Cardiology, Vol. 108, No. 5, 2011, pp. 682-690. doi:10.1016/j.amjcard.2011.04.015

[15] M. Suzukawa, M. Abbey, P. R. Howe and P. J. Nestel, "Effects of Fish Oil Fatty Acids on Low Density Lipoprotein Size, Oxidizability, and Uptake by Macrophages," Journal of Lipid Research, Vol. 36, No. 3,1995, pp. 474-484.

[16] C. D. Gardner, S. P. Fortmann and R. M. Kraus, "Association of Small Low-Density Lipoprotein Particles with the Incidence of Coronary Heart Disease in Men and Women," Journal of the American Medical Association, Vol. 276, No. 11, 1996, pp. 875-881. doi:10.1001/jama.1996.03540110029028

[17] L. J. Schwellenbach, K. L. Olson, K. J. McConnell, R. S. Stolcpart, J. D. Nash, J. A. Merenich and Clinical Pharmacy Cardiac Risk Service Study, "The TriglycerideLowering Effects of a Modest Dose of Docosahexaenoic Acid Alone Versus in Combination with Low Dose Eicosapentaenoic Acid in Patients with Coronary Heart Disease and Elevated Triglycerides," Journal of the American College of Nutrition, Vol. 25, No. 6, 2006, pp. 480-485.

[18] C. M. Ballantyne, A. Corsini, M. H. Davidson, H. Holdaas, T. A. Jacobson, H. Holdaas, et al., "Risk for Myopathy with Statin Therapy in High-Risk Patients," Archives of Internal Medicine, Vol. 163, No. 5, 2003, pp. 553-564. doi:10.1001/archinte.163.5.553

[19] D. J. Graham, J. A. Staffa, D. Shatin, S. E. Andrade, S. D. Schech, L. La Grenade, et al., "Incidence of Hospitalized Rhabdomyolysis in Patients Treated with Lipid-Lowering Drugs," Journal of the American Medical Association, Vol. 292, No. 21, 2004, pp. 2585-2590. doi:10.1001/jama.292.21.2585 